\title{
O TRATAMENTO DO MEDO DE ALTURAS
}

\author{
Acrophobia treatment
}

\author{
Carlos Magalhães Coelho ${ }^{a}$, Margarida Pocinho ${ }^{\mathrm{b}}$, Carlos Fernandes da Silva ${ }^{\mathrm{c}}$
}

\footnotetext{
a School of Human Movement Studies, University of Queensland, Australia Research Fellow, e-mail: ccoelho@hms.uq.edu.au

${ }^{b}$ Professora Doutora da Universidade da Madeira, Portugal, e-mail: margarida.pocinho@madeira-edu.pt

c Professor Doutor da Universidade de Aveiro, Portugal, e-mail:csilva@dce.ua.pt
}

\begin{abstract}
Resumo
Neste artigo, mencionamos as estratégias terapêuticas que julgamos mais apropriadas ao tratamento do medo de alturas. Comparamos vários estudos que usaram várias técnicas e referimos a nossa experiência clínica e de investigação. Considerámos, igualmente, os novos tratamentos que usam ambientes de realidade virtual para o tratamento da acrofobia. Actualmente a realidade virtual (RV) é utilizada pelos governos, indústrias, academias e investigadores individuais, favorecendo uma multiplicidade de possíveis produtos e aplicações em diversas áreas. Estes sistemas têm igualmente inúmeras aplicações no campo da psicologia, por exemplo na avaliação e tratamento de desordens alimentares e diversas fobias, como medo de aranhas, medo de andar de avião e claustrofobia. Concluímos que, até o momento presente, a prática reforçada de Leitenberg (1976) é o tratamento mais bem estudado para a acrofobia. O objectivo principal deste tratamento de fobias é a diminuição do evitamento do estímulo ou situação temida; a moldagem de comportamentos de aproximação apropriados. $\mathrm{O}$ terapeuta providencia ao cliente uma exposição gradual ao alvo ameaçador e reforça melhorias sucessivas à habilidade do cliente para interagir com o estímulo alvo. A duração das exposições pode ser baseada numa duração de tempo ou num número de apresentações práticas.
\end{abstract}

Palavras-chave: Acrofobia; Medo de alturas; Tratamento. 


\begin{abstract}
In this article we refer the therapeutic strategies we think more appropriates to the treatment of fear of heights. We compare several studies that use this technique and refer our own experience clinical and investigational experience. We also consider the more recent treatments that use virtual reality environments in the treatment of acrophobia. Nowadays, virtual reality $(V R)$ is used by governments, industries, the academic world and individual researchers, fostering a multiplicity of possible products and applications in areas such as business, entertainment, medicine and health care. These VR systems also have countless applications in the Psychology field, as in the assessment and treatment of food disorders, spider phobia, fear of flying and claustrophobia. We conclude that the reinforced practice (Leitenberg, 1976) is the best studied treatment for acrophobic patients. The main goal of this phobia treatment is to model appropriate coping behaviours, through a process of successive approaches. As such, the therapist promotes approach behaviours, reinforcing advances and removing the negative reinforcement of avoidance. The therapist moulds the desired approaches, offering the client a gradual exposure to the threatening target and reinforcing successive improvements in the client's ability to interact with, or remain exposed to, the targeted stimulus. The duration of exposures may be based on a length of time or on a number of practical presentations.
\end{abstract}

Keywords: Acrophobia, Fear of heights, Treatment.

\section{INTRODUÇÃO}

A história do tratamento das fobias específicas tem a idade da história das terapias comportamentais (O’brien, 1981). De facto, já no famoso estudo de Watson e Rayner, de 1920, os autores terminam o seu artigo com algumas sugestões para o tratamento das fobias, ou através da estimulação de zonas erógenas ou pela alimentação, emparelhada com o estímulo fóbico. Mais tarde, com Mowrer (1939), surge a teoria dos dois factores, que se manteve praticamente inalterada até à actualidade. Essencialmente, esta teoria constata que a ansiedade fóbica é uma resposta condicionada, desencadeada pelo estímulo condicionado (a situação fóbica). Adiciona porém, que o evitamento da situação desenvolve-se e mantém-se por ser reforçada pela redução da ansiedade (reforço negativo). Este evitamento com sucesso da situação fóbica assegura a conservação da ansiedade, perpetuando a perturbação. De acordo com a teoria dos dois factores de Mowrer, o condicionamento clássico é responsável pela aquisição do medo e o condicionamento operante pela aprendizagem do comportamento de evitamento (Emmelkamp, Bouman \& Scholing, 1989). Actualmente é praticamente consensual a ideia de que o facto do evitamento diminuir a ansiedade no momento, seja este o responsável pela manutenção do medo (Agras \& Jacob, 1981; Arntz, Rauner e Van Den Hout, 1995; Tolin, Lohr, Lee \& Sawchuk, 1999).

Mas foi uma técnica denominada de dessensibilização sistemática, de Wolpe (1958), o marco principal no desenvolvimento de tratamentos eficazes das fobias. Wolpe baseia-se na teoria de Mowrer e, através de uma técnica engenhosa, faz com que os seus pacientes evitem o evitamento, ou seja, permaneçam perante o estímulo temido. No seu livro "Psicoterapia por inibição recíproca", Wolpe explica que este procedimento consiste em emparelhar situações temidas imaginariamente com relaxamento muscular, o qual serve como resposta inibidora de ansiedade. Central para o desenvolvimento deste procedimento estava a noção de que o relaxamento muscular profundo era um antagonista da ansiedade. Em virtude de as cenas imaginadas serem ordenadas de forma gradual, de forma a provocar níveis mínimos de ansiedade, Wolpe propôs que o relaxamento poderia ser capaz de suprimir as propriedades ansiógenas do estímulo, quebrando o laço entre estes.

Uma série de estudos posteriores indicaram que a dessensibilização sistemática era eficaz na eliminação de medos e, na sua maioria, mais eficaz que outras terapias (Mavissakalian \& Barlow, 1981). A demonstração da eficácia deste procedimento foi 
extremamente importante na história da investigação terapêutica. Mas, em nossa opinião, mais importante para a investigação na redução do medo, foram as questões levantadas acerca dos mecanismos de acção da dessensibilização e o início da avaliação de tratamentos alternativos para o tratamento dos medos e fobias, tais como, a imersão imaginada, a prática reforçada e a modelagem.

Após Wolpe, inúmeros estudos visaram a procura dos ingredientes terapêuticos activos, e actualmente, a exposição é considerada o ingrediente terapêutico de maior importância no tratamento das fobias (e.g., Marks, 1987; Öhman, 1996; Capafons, 2001; Öhman \& Mineka, 2001). De todas as formas que estudámos, julgamos que a prática reforçada (Leitenberg, 1976) é, contudo, a forma terapêutica que melhor associa os vários componentes terapêuticos para o tratamento das fobias. Iremos a seguir debruçarmo-nos sobre este tratamento, baseado numa abordagem operante da redução do medo.

\section{PRÁTICA REFORÇADA}

Os indivíduos fóbicos parecem escapar dos seus medos demasiado depressa para poderem aprender que o seu desconforto desaparece (Marks, 1987). A prática reforçada (Leitenberg, 1976) assume que as melhorias nos componentes cognitivos e fisiológicos da ansiedade ocorrerão após a moldagem de respostas de aproximação e da prática continuada destas respostas. Note-se que Leitenberg considera já os componentes cognitivos da exposição, e não apenas os fisiológicos, como fazia Wolpe. É também de sublinhar que Leitenberg reúne estudos seus e de outros investigadores no sentido de isolar variáveis terapêuticas, com a intenção de avaliar a sua validade (e.g., Leitenberg, Agras, Edwards, Thomson \& Wincze 1970; Agras \& Jacob, 1981; Leitenberg, Agras, Allen, Butz, \& Edwards, 1975).

Desta forma, os resultados das investigações de Leitenberg et al. (1970) indicaram que as melhorias ocorriam durante os períodos de prática ao vivo, e estudos adicionais (e.g., Agras \& Jacob, 1981; Leitenberg et al.1975) sugeriram que variáveis como o elogio, o feedback preciso acerca das melhorias, o conhecimento do desempenho e as expectativas do terapeuta eram também ingredientes terapêuticos importantes. Estes ingredientes foram posteriormente combinados num modelo terapêutico designado de prática reforçada (Leitenberg, 1976), e a sua combinação foi testada relativamente a outras alternativas de tratamento para fobias. Esta estratégia de tratamento consiste numa combinação de ingredientes terapêuticos, incluindo a exposição aos estímulos desencadeadores de medo, instruções terapêuticas, controlo do progresso do cliente, feedback acerca do desempenho e reforço contingente do desempenho (Leitenberg, 1976, O’brien, 1981).

O objectivo principal deste tratamento de fobias é a moldagem de comportamentos de aproximação apropriados, por um processo de aproximações sucessivas. O tratamento é conseguido através da diminuição da fuga da situação temida que, como já referimos, parece ser o elemento perpetuador do medo. Supõe-se que a ausência de consequências terá como resultado a extinção do medo, sendo este o ingrediente comum das terapias de exposição. Como tal, o terapeuta fomenta comportamentos de aproximação, reforçando as aproximações e removendo o reforço negativo do evitamento. O terapeuta molda as aproximações desejadas, providenciando ao cliente uma exposição gradual ao alvo ameaçador e reforçando melhorias sucessivas à habilidade do cliente para interagir com, ou permanecer exposto ao estímulo-alvo. A duração das exposições pode ser baseada numa duração de tempo (geralmente de 15-30 minutos) ou num número de apresentações práticas.

Sendo o feedback sobre o desempenho e o reforço contingente ingredientes principais da prática reforçada, o desempenho do cliente deverá serquantificado apropriadamente. O terapeuta pode medir o progresso anotando, por exemplo, a proximidade do cliente ao estímulo-alvo, a distância atravessada, ou número de itens da hierarquia completados com sucesso. O feedback do desempenho é providenciado ao cliente durante a exposição. Leitenberg et al. (1975), numa série de experiências de caso-único, demonstraram que o feedback do desempenho é um ingrediente importante da prática reforçada. Os sujeitos fóbicos demonstraram pouca melhoria quando o feedback foi omitido dos procedimentos terapêuticos. Quando o feedback era incluído no tratamento, todos os sujeitos melhoraram, alguns deles de forma bastante significativa. Quando o feedback foi de novo omitido, contudo, o desempenho não se deteriorou. Assim, 
o feedback de desempenho parece particularmente importante no início do tratamento. Estes investigadores especularam que o feedback pode ser importante, dado lembrar o cliente dos objectivos específicos do tratamento e permitir a identificação de ligeiras melhorias que, de outra forma, poderiam não ser reconhecidas. Este último efeito pode, conseqüentemente, aumentar a motivação do cliente para tentar persistir nas aproximações ao estímuloalvo e aumentar as expectativas relativamente aos resultados do tratamento.

\section{EFEITOS COGNITIVOS DA EXPOSIÇÃO}

O primeiro estudo relativo aos processos cognitivos implicados na acrofobia foi realizado por Sutton-Simon e Goldfried (1979). Os autores compararam o pensamento irracional e autoverbalizações negativas de acrofóbicos e fóbicos sociais $(n=60)$. Apesar de terem encontrado crenças irracionais marcadas em ambos os grupos, apenas os acrofóbicos tendiam a emitir constatações negativas acerca de si. Os autores crêem que a emissão de constatações negativas acerca de si é situacionalmente específica, surgindo apenas em conjunto com relatos de desconforto ou stress. Este estudo é importante na medida em que sugere uma ausência de um "neuroticismo" geral subjacente aos sujeitos com acrofobia. Mas, será pertinente para o clínico observar se o desconforto surge apenas em circunstâncias relativas a alturas ou se surge igualmente em outras situações, tais como acontece em sujeitos com agorafobia que, como se sabe, tem habitualmente associado níveis mais elevados de ansiedade traço (Antony \& Barlow, 1996).

Posteriormente, Williams e Watson (1985) examinaram o pensamento irracional em termos de perigo percebido durante uma tarefa de desempenho. Eles notaram que entre os sujeitos que referiam medo, os que completaram o exame comportamental diferiam dos que não o fizeram, em particular, no que diz respeito a pensamentos catastróficos, confirmando os dados de Sutton-Simon e Goldfried (1979) acerca da natureza do pensamento dos acrofóbicos. Marshall, Bristol e Barbaree (1992) corroboram a ideia de que muitos sujeitos com acrofobia têm crenças irracionais acerca de consequências catastróficas que podem surgir quando entram num local elevado. Eles acreditam, e repetem para si próprios, que, por exemplo, a estrutura irá colapsar, um forte vento subitamente irá soprar e levá-los do prédio, ou que, acidentalmente, cairão da varanda de um teatro. Estes pensamentos, claramente irracionais, apresentam-se persistentemente em locais altos, e são igualmente repetidos sub-vocalmente por muitos dos sujeitos quando entram numa situação fóbica.

Estes três estudos parecem apontar no sentido da existência de cognições de natureza catastrófica, por parte dos sujeitos com acrofobia, em particular durante os momentos de exposição. Mas, apesar da aparente importância fenomenológica das cognições desadaptativas nas fobias simples, autores como Last (1987), Marks (1987) Öhman (1996) e Öhman e Mineka (2001) não apoiam o uso de tratamentos cognitivos com esta população, quando se compara com intervenções comportamentais. Mas a necessidade de exposição, não significa que não se operem alterações cognitivas durante a mesma. De facto, a exposição directa parece providenciar uma via para obter dados de infirmação e de criação de interpretações baseadas na evidência. Por exemplo, pessoas que acreditam que podem cair de uma varanda aprendem que não caiem quando permanecem na mesma (Craske \& Deborah, 2001). Acredita-se pois, que a redução do medo é dependente da formação de novas associações entre os estímulos, respostas e significados.

Öhman e Mineka (2001) referem que as cognições são importantes nas fobias essencialmente para a manutenção do comportamento fóbico. As expectativas, crenças de controlo e interpretações catastróficas têm um papel importante na ansiedade e nos ataques de pânico. A ausência de controlo percebido numa situação potencialmente aversiva gera medo, enquanto a aquisição de um sentido de mestria o reduz. Quanto mais informação temos acerca do grau de aversão esperado, a sua probabilidade de ocorrência, a sua duração provável e os seus sinais premonitórios, maior a probabilidade de encontrarmos formas de prevenir ou reduzir as consequências de algum tipo de consequência (Rachman, 1990). Mas a melhor forma de potenciar a obtenção de controlo e informação parece ser através da exposição, e as pistas de medo estão frequentemente ausentes quando são avaliadas de forma lógica, relativamente aos erros de julgamento, no gabinete do terapêuta (Craske \& Deborah, 2001). 
Thorpe e Salkovskis (1995, 1998) e Salkovskis, Clark, Hackmann, Wells e Gelder (1999) defendem que a mudança nas crenças, mais do que a habituação a um estímulo repetido, é o principal mecanismo que medeia a eficácia da exposição. Há, por isso, um bom senso em focar esforços terapêuticos nestes processos, em sujeitos com ansiedade. Mas, para Öhman (1996), esses esforços são insuficientes porque falham determinantes da ansiedade que permanecem inacessíveis à consciência. Alguns dos mais importantes processos psicológicos relativos ao medo e a ansiedade permanecem inconscientes, na medida em que operam fora do foco da atenção consciente e controlada. Por exemplo, uma resposta fóbica parece estar organizada a um nível inconsciente, sendo os componentes da resposta, iniciados antes do estímulo desencadeador estar representado na consciência. Como tal, para o sucesso do tratamento Öhman e Mineka (2001) salientam que é necessário que o sujeito com fobia se confronte com o estímulo temido, de forma a extinguir a activação automática do medo.

Este debate entre os aspectos cognitivos ou de habituação como determinantes principais na extinção do medo não é recente. Os factores cognitivos parecem mudar radicalmente antes e depois do tratamento. Mas uma das maiores dificuldades com o tratamento de exposição é que mesmo os seus proponentes mais ardentes não estão certos de porquê que funciona (Mavissakalian \& Barlow, 1981).

Dado a especificidade do medo de alturas iremos a seguir centrar-nos nessa fobia. Vamos tentar dar a perceber algumas das particularidades da desordem e do seu tratamento.

\section{O TRATAMENTO DA ACROFOBIA}

Os estudos que a seguir referimos versam elementos variáveis tais como: 1) a comparação entre um tratamento quando aplicado por um terapeuta e a mesma intervenção aplicada pelo próprio; 2) a comparação entre o modelo de exposição, e o modelo de auto eficácia; 3) a duração do tratamento; 4) o efeito do uso comentários positivos por parte do participante (self-statements), entre outros.

Salientaremos os estudos de Williams, Dooseman e Kleinfield (1984) e de Williams, Turner e Peer (1985), dado apresentarem algumas semelhanças com a nossa experiência clínica e de investigação, que por fim referiremos.

Baker, Cohen e Saunders (1973) compararam dois tipos de tratamento aplicado a acrofobia. A dessensibilização sistemática aplicada por um terapeuta e a mesma intervenção aplicada pelo próprio participante com a ajuda de um gravador com as instruções para a dessensibilização. Os resultados das intervenções foram igualmente eficazes. Mantiveram-se os ganhos numa avaliação de follow-up após 8 meses no tratamento com terapeuta e, curiosamente, foram notados ganhos adicionais no follow-up dos sujeitos que usaram o tratamento por gravação. Os resultados sugerem que a dessensibilização é um tratamento eficaz mesmo com um contacto terapêutico reduzido.

Os autores notam que existem pessoas que funcionam melhor numa situação terapêutica que lhes proporcione uma grande autonomia e controlo sobre os procedimentos usados. Adicionalmente, os sujeitos "do gravador" podem ter assumido uma responsabilidade contínua em ajudar-se a si próprios após o termo formal do tratamento dado que este requereu mais responsabilidade pessoal do sujeito participante do que do sujeito terapêuta (Baker, Cohen \& Saunders, 1973).

Mas o estudo de Williams et al. (1984), ao contrário do anterior, de Baker et al. (1973), dá muita importância ao papel do terapeuta no tratamento do medo de alturas. Os autores comparam o modelo de exposição e o modelo de auto eficácia em sujeitos com medo severo de alturas e de conduzir. O modelo de auto eficácia foi significativamente mais eficaz que o modelo de exposição em relação ao comportamento, sentimento de auto eficácia e da diminuição da ansiedade antecipatória e ansiedade durante o desempenho (Williams et al. 1984). Ao contrário do modelo de prática reforçada de Leitenberg (1976), a ênfase do tratamento de auto eficácia não é colocado na exposição. Este modelo salienta como factores primordiais a qualidade e quantidade de informação para o aumento da eficácia que as pessoas adquirem e a forma como as pessoas integram essa informação no julgamento das suas capacidades.

Mas a metodologia de Williams e colaboradores (1984) parece-nos criticável. É de salientar que, durante o tratamento de exposição, o terapeuta observava os participantes desde o 
solo. Se os sujeitos descessem antes do final do tratamento (que durava 60 minutos), eram lembrados que o tempo mantido no solo não contava na duração do tratamento. Julgamos que este procedimento pode ter servido como uma forma de punição do evitamento. Adicionalmente, o terapeuta que aplicou o tratamento de exposição nunca acompanhou os sujeitos ao prédio, nem providenciou assistência adicional. Já o tratamento de mestria contou com inúmeras técnicas adicionais. Por exemplo: 1) inicialmente o terapeuta acompanhou o participante, retirandose mais tarde; 2) identificou e impediu comportamentos defensivos como, por exemplo, o "congelar" e o evitar olhar; 3) identificou com o cliente momentos, posturas e locais onde olha de maior ansiedade; 4) permitiu que o cliente variasse o olhar e as posturas, como o andar ou ficar parado, mas sempre encorajando a estar confronto com a situação temida; 5) em momentos de maior segurança o terapeuta entra para a sala onde se encontra a varanda de forma a deixar o participante só, e depois regressa e reavalia, elogiando os progressos; 6) o terapeuta manteve-se vigilante corrigindo as acções do cliente de forma a assegurar que os sujeitos aproveitassem a situação e potenciar os efeitos terapêuticos da exposição.

Este método é semelhante ao da prática reforçada, e tendo em conta a metodologia aplicada, não é de admirar que tenha havido uma notória diferença de resultados entre os tratamentos.

Num semelhante estudo posterior, Williams et al. (1985) comparam o modelo da dessensibilização sistemática e o modelo de autoeficácia em sujeitos com acrofobia severa. Os autores concluem de novo que o modelo de auto-eficácia é mais eficaz que o modelo de dessensibilização no tratamento de sujeitos com acrofobia, tanto a nível de resultados comportamentais, como na melhoria de percepções de auto eficácia e na redução de ansiedade antecipatória e pensamentos de perigo. De novo, este tratamento contou com a mestria de sub-tarefas; atingir objectivos intermédios; suporte físico (segurar no braço do cliente); modelagem (demonstração); eliminação de manobras defensivas (e.g., rigidez, ou ficar parado), e desempenhos variados (Williams et al. 1985).

Apesar de nos parecerem parciais nas suas metodologias, estes estudos foram importantes na medida em que alertam para o perigo de o terapeuta, no modelo de dessensibilização, poder ser visto como um mero executor do processo de extinção que se produz principalmente pela passagem do tempo.

Outros estudos relativos ao tratamento comportamental de fobias centraram-se no tempo e frequência dos tratamentos de exposição. De forma geral, concluem que uma exposição continuada e longa é melhor que uma exposição curta e intermitente (Marshall 1985; Marks, 1987). Apesar dos trabalhos de Ost (e.g., Ost, 1989; Hellstrom \& Ost, 1996) serem conhecidos pelo seu sucesso com exposições únicas, as mesmas são de duração prolongada, de 60 a 180 minutos. Relativamente a acrofobia, um artigo interessante de Marshall (1985) refere dois estudos onde se avaliam os efeitos da exposição ao vivo, em sujeitos com medo de alturas, consoante a duração do tratamento. O estudo avaliou, igualmente, o efeito do uso de comentários para si próprios (self-statements) de forma a lidar com a situação. No primeiro estudo, a duração total da exposição foi ignorada e os sujeitos apenas se expuseram até que a ansiedade regressasse a linha de base. No segundo estudo o tempo total de exposição permaneceu constante, variando as exposições, ora de forma intermitente, por períodos breves, ou de forma continuada, até que a linha de base fosse atingida. As exposições breves resultaram em pouca ou nenhuma mudança, enquanto os outros procedimentos reduziram o medo. A proposta de que as exposições breves aumentam o medo é concordante com outras tais como a teoria da incubação de Eysenck (1968), que sugere que as exposições breves inclusive provocarão um aumento do medo.

Emmelkamp, Boumann e Scholing (1989), e Rachman (1990) põem em causa esta ideia, dado relatarem estudos seus, nos quais permitiram aos participantes a retirada da situação fóbica, mantendo-se contudo os ganhos terapêuticos. Marshall (1985) critica alguns destes estudos por usarem um número reduzido de sujeitos e de categorizarem os resultados dicotomicamente como "melhorados" ou "não melhorados", podendo melhorias pequenas ser consideradas como significativas. Adicionalmente, julgamos que uma excessiva brevidade na exposição pode ter efeitos semelhantes aos do evitamento, perpetuando o medo, como referimos anteriormente. Assim, de uma forma geral, os estudos suportam a ideia de que a ansiedade deve ser reduzida a níveis mínimos 
antes da exposição terminar, sendo mais eficazes as exposições "longas" que as "breves".

É de salientar, relativamente aos uso de comentários positivos, que a reestruturação cognitiva parece ter um impacto positivo maior quando o sujeito apresenta níveis de ansiedade mais baixos, podendo inclusive ser distractiva quando a ansiedade é elevada, minimizando os ganhos terapêuticos (Marshall, 1985). Esta descoberta faz pensar que, em terapia, existem momentos oportunos para se trabalhar diferentes aspectos com os clientes.

A este propósito, Abelson e Curtis (1989) notam que os investigadores da ansiedade a medem usualmente em termos de "sistemas" de resposta subjectiva, comportamental e fisiológica. Esta "trilogia" nasce com Lang (1993), que considera que a ansiedade se pode exprimir através de um sistema orgânico parcialmente independente: o comportamento verbal; a activação fisiológica e o desempenho motor. Num dado indivíduo, a ansiedade pode ser predominantemente cognitiva, noutro pode ser vegetativa e noutro mais motora. E a mesma pessoa pode ter manifestações cognitivas e vegetativas elevadas e, não obstante, aproximarse da situação temida. Ou, referir que não tem medo, mas evitar marcadamente o estímulo. Rachman (1990) refere que os três sistemas não são modificáveis ao mesmo tempo. O componente motor é mais influenciável, pois facilmente se podem criar situações de confronto com o estímulo. O componente cognitivo é o último a ser corrigido, dado que as modificações das expectativas de eficácia pessoal exigirem um longo tempo para correcção.

As medidas entre estes sistemas frequentemente variam independentemente umas das outras durante o curso dos tratamentos, um fenómeno que tem sido apelidado de dessincronia. Os resultados do seu estudo em dois sujeitos com acrofobia, suportam a ideia de que o sistema fisiológico não é um sistema sincronizado único, pelo que a sincronia entre os 3 sistemas não é necessária para um tratamento de uma fobia ou para a preservação dos ganhos em follow-up.

Por fim, julgamos importante referir um estudo de Schneider (1982), que podemos considerar um percursor da aplicação de sistemas virtuais ao tratamento do medo de alturas (Botella, Baños, Perpiña, Villa, Alcañiz, \& Rey, 1998). No seu artigo, Schneider (1982) nota que a dessensibilização ao vivo do medo de alturas pode ser problemática dado as exposições apropriadas à prática que sejam seguras e acessíveis para fins terapêuticos, podem ser inconvenientes ou pouco práticos de se conseguir. Assim, o autor usou binóculos com lentes invertidas para alterar a percepção de profundidade, de forma a magnificar a sensação de altura durante um processo de exposição ao vivo. Ao olhar por um $8^{\circ}$ andar, por exemplo, o sujeito tinha uma altura aparente de 56 andares. Tratou-se de um estudo de caso: o sujeito era um homem de 40 anos, casado, que vivia numa cidade repleta de arranha-céus. Assim, ao olhar por um $8^{\circ}$ andar o sujeito tinha uma altura aparente de 56 andares: "a retirada dos binóculos ao fim de cada visita produzia uma diminuição dramática da altura aparente" (Schneider, 1982, p. 334). O folow-up, realizado oito meses após o fim da intervenção, avaliou uma manutenção dos efeitos do tratamento, e curiosamente, os ganhos do tratamento generalizaram-se relativamente ao medo de voar que este participante também tinha.

Actualmente a realidade virtual (RV) é utilizada pelos governos, indústrias, academias e investigadores individuais, favorecendo uma multiplicidade de possíveis produtos e aplicações em diversas áreas. Estes sistemas de realidade virtual têm igualmente inúmeras aplicações no campo da psicologia, por exemplo na avaliação e tratamento de desordens alimentares (Riva, 1997) e diversas fobias, como medo de aranhas (Carlin, Hofman, \& Weghorst, 1997), medo de andar de avião (North, North \& Coble, 1996; Rothbaum, Hodges, Watson, Kessler, \& Opdyke, 1996) e claustrofobia (Botella et al., 1998).

Sabendo que a exposição ao vivo é eficaz para indivíduos com medo de alturas (e.g., Abelson \& Curtis, 1989; Emmelkamp \& Felten, 1985; Williams, Dooseman \& Kleinfield, 1984; Williams, Turner \& Peer, 1985), realizamos um estudo recente onde investigamos se esse tipo de tratamento se pode efectuar por intermédio de sistemas de realidade virtual (Coelho, Silva, Santos \& Silvério 2005). Através de uma metodologia de caso único, o participante, após 3 sessões de exposição a alturas simuladas por um sistema de realidade virtual, apresenta progressos significativos nas medidas de ansiedade, evitamento e medidas comportamentais, quando confrontado com ambientes de alturas reais. É de salientar que a componente de exposição usada no estudo foi a prática reforçada de Leitenberg (1976). 


\section{CONSIDERAÇÕES FINAIS}

Parece existir um ganho acrescido quando se integram várias técnicas numa intenção terapêutica. A exposição, modelagem, informação e feedback positivo são úteis, e parecem ser os principais ingredientes de um tratamento de sucesso numa terapia para uma fobia simples. Mas os tratamentos que aplicam uma técnica exclusiva, sem o apoio de qualquer outra, como no estudo de Williams, Dooseman e Kleinfield (1984), os ganhos terapêuticos parecem reduzidos. Como notámos antes, durante o tratamento de exposição, o terapeuta observava os participantes desde o solo, e ao invés, no tratamento por modelagem, existia igualmente bastante feedback. Num estudo de Menzies e Clarke (1993), numa comparação de tratamentos de fobia a água, apresentam-se resultados inversos aos de Williams et al. (1984). Os autores observaram que o tratamento ao vivo produziu ganhos clínicos e estatísticos significativos que se generalizaram a outras situações envolvendo água e que se mantiveram durante um período de três meses de follow up. O tratamento vicariante (com um modelo de mestria) não obteve resultados estatísticos significativos maiores que os observados no grupo de controlo.

Parece-nos, pois, que um tratamento de exposição ideal deverá ter um componente informativo, de observação, de prática e de feedback de progresso. Esse tratamento encontra-se bem fundamentado na prática reforçada de Leitenberg (1976), e esse parece ser o mais adequado a usar em indivíduos com acrofobia, quer em ambientes reais, quer em ambientes de realidade virtual.

\section{REFERÊNCIAS}

Abelson, J. L., \& Curtis, G. C. (1989). Cardiac and neuroendocrine responses to exposure therapy in height phobics: Desyncrony within the "physiological response system" Behaviour Research \& Therapy, 27(5), 561-567.

Agras, W. S.; Jacob, R. G. (1981). Phobia: Nature and Measurement. In M, Matig, \& D, H., Barlow. Phobia: Psychological and pharmacological treatment. New York: The Guilford Press.
Antony, M. M., Barlow, D. H. (1996). Emotion theory as a framework for explaning panic attacks and panic disorders. In R. M. Rapee (Ed.) Current Controversies in the Anxiety Disorders. (pp.55-76). London. Guilford Press.

Arntz, A., Rauner, M., \& Van Den Hout, M. (1995). "If I feel anxious, there must be danger": ex-consequentia reasoning in inferring danger in anxiety disorders. Behaviour Research and Therapy, 33(8), 917-925.

Baker, B. L., Cohen, D. C., \& Saunders, J. T. (1973). Self-directed desensitization for acrophobia. Behaviour Research and Therapy, 11, 79-89.

Botella, C., Baños, R.M., Perpiña, C., Villa, H., Alcañiz, M., \& Rey, A. (1998). Virtual reality treatment of claustrophobia: A case report. Behaviour Research and Therapy, 36, 239-246.

Capafons, J. I. (2001). Tratamientos psicológicos eficaces para las fobias específicas. Psicothema, 13, 447-452.

Carlin, A., Hoffman, H., \& Weghorst, S. (1997). Virtual reality and tactile augmentation in the treatment of spider phobia: A case report. Behaviour Research and Therapy, 35, 153-158.

Coelho, C. S. M., Silva, C. F., Santos, J. A, Silvério, J. A. S. (2005). Realidade Virtual Aplicada ao tratamento da acrofobia: Estudo de Caso. Psiquiatria Clínica, 26(2), 153-165.

Craske, M. G., Deborah, C. P (2001). Cognitive biases in anxiety disorders and their effect on cognitive-behavioral treatment. Bulletin of the Menninger Clinic. 65(1), 58-77.

Emmelkamp, P. M., Bouman, T. K., Scholing, A. (1989). Anxiety disorders: A practitioners guide. Chichester: John Wiley \& Sons.

Emmelkamp, P. M., \& Felten, M. (1985). The process of exposure in vivo: Cognitive and physiological changes during treatment of acrophobia. Behaviour Research \& Therapy, 23, 219-223. 
Eysenck, H. J. (1968). A theory of the incubation of anxiety/fear responses. Behaviour Research and Therapy, 6, 309-321.

Hellstrom, K; Ost, L.-G. (1996). Prediction of outcome in the treatment of specific phobia. A cross validation study. Behaviour Research and Therapy, 34(5/6), 403-411.

Lang, P. J. (1993). The three-System Approach to Emotion. In N. Birbaumer, \& A. Ohman. (Ed.). The Structure of Emotion. (pp. 1830). Seattle: Hogrefe \& Huber Publishers.

Last, C. G. (1987). Simple Phobias. In L. Michelson \& L. M. Asher (Ed.). Anxiety and Stress Disorders. Cognitive-Behavioral Assessment and Treatment. (pp.176-190). New York: The Guilford Press.

Leitenberg, H. (1976). Behavioral approaches to treatment of neuroses. In H. Leitenberg (Ed.). Handbook of behavior modification and behavior therapy. Englewood Cliffs: Prentice-Hall.

Leitenberg, H., Agras, S., Allen, R., Butz, R., \& Edwards, J. (1975). Feedback and therapist praise during treatment of phobia. Journal of Consulting and Clinical Psychology, 43, 396-404.

Leitenberg, H., Agras, S., Edwards, J. A., Thomson, L. E., \& Wincze, J. P. (1970). Practice as a psychotherapeutic variable: an experimental analysis within single cases. Journal of Psychiatric Research, 7, 215-225.

Marks, I. M. (1987). Fears, fobias and rituals. panic, anxiety and their disorders. Oxford. Oxford University Press.

Marshall, W. L. (1985). The effects of variable exposure to flooding therapy. Behavior Therapy, 16, 117-135.

Marshall, W. L., Bristol, D., Barbaree, H. E. (1992). Cognitions and courage in the avoidance behavior of acrophobics. Behaviour Research and Therapy, 30(5), 463-470.

Mavissakalian, M., Barlow, D. H. (1981). Phobia: an overview. In: M., Mavissakalian, \& D. H. Barlow. Phobia: Psychological and pharmacological treatment. The Guilford Press: New York.
Menzies, R. G., Clarke, J. C. (1993). A comparison of in vivo and vicarious exposure in the treatment of childhood water phobia. Behaviour Research and Therapy, 31 (1), 9-15.

Mowrer, O. H. (1939). A stimulus-response analysis of anxiety and its role as a reinforcing agent. Psychological Review, 46, 553-565.

North, M. M., North, S. M., \& Coble, J. R. (1996). Virtual reality therapy: An innovative paradigm. Colorado: IPI Press.

O'brien, G. T. (1981). Clinical treatment of specific phobias. In M. Mavissakalian, \& D. H. Barlow (Ed.). Phobia: Psychological and Pharmacological Treatment. (pp. 63102). New York: The Guilford Press.

Öhman, A. (1996). Preferential preattentive processing of threat in anxiety: preparedness and attentional biases. In R. M. Rapee (Ed.). Current controversies in the anxiety disorders. (pp. 253-290). London: Guilford Press.

Öhman, A., Mineka, S. (2001). Fears, fobias, and preparedness: toward an evolved module of fear and fear learning. Psychological Review, 108(3), 483-522.

Ost, L.-G. (1989). One-session treatment for specific phobias. Behaviour Research and Therapy, 27, 1-7.

Rachman, S. J. (1990). Fear and Courage. New York: Freeman and Company.

Riva, G. (1997). The virtual environment for body-image modification (VEBIM): Development and preliminary evaluation. Presence: Teleoperators \& Virtual Environments, 6, 106-118.

Rothbaum, B., Hodges, L., Watson, B., Kessler, G., \& Opdyke, D. (1996). Virtual reality exposure therapy in the treatment of fear of flying: a case report. Behaviour Research and Therapy, 34, 477-481.

Salkovskis, P. M., Clark, D. M., Hackmann, A., Wells, A., \& Gelder, M. G. (1999). An experimental investigation of the role of safetyseeking behaviours in the maintenance of panic disorder with agoraphobia. Behaviour Research and Therapy, 37, 559-574. 
Schneider, J. W. (1982). Lens-assisted in vivo desensitization to heights. Journal of Behaviour \& Experimental Psychiatry, 13, 333-336.

Sutton-Simon, K., \& Goldfried, M. R. (1979). Faulty thinking patterns in two types of anxiety. Cognitive Therapy and Research, 3, 193-203.

Thorpe, S. J., \& Salkovskis, P. M. (1995). Phobic beliefs: do cognitive factors play a role in specific phobias? Behaviour Research and Therapy, 33, 805-816.

Thorpe, S. J., \& Salkovskis, P. M. (1998). Selective attention to real phobic and safety stimuly. Behaviour Research and Therapy, 36, 471-481.

Tolin, D. F., Lohr, J. M., Lee, T. C., \& Sawchuk, C. N. (1999). Visual avoidance in specific phobia. Behaviour Research and Therapy, 37, 63-70.

Watson, J. B., Rayner, R. (1920). Conditioned emotional reactions. Journal of Experimental Psychology, 3, 1-14.
Williams, S. L., \& Watson, N. (1985). Perceived danger and perceived self-efficacy as cognitive determinants of acrophobic behavior. Behavior Therapy, 16, 136-146.

Williams, S. L., Dooseman, G. \& Kleinfield, E. (1984). Comparative effectiveness of guided mastery and exposure treatments for intractable phobias. Journal of Consulting and Clinical Psychology, 52, 505-518.

Williams, S. L., Turner, S. M., Peer, D. F. (1985). Guided Mastery and Performance Desensitization Treatments for Severe Acrophobia. Journal of Consulting and Clinical Psychology, 53, 237-247.

Wolpe, J. (1958). Psychotherapy and reciprocal inhibition. Stanford: Stanford University Press.
Recebido: 03/12/2007

Received: 12/03/2007

Aprovado: 03/03/2008

Approved: 03/03/2008 